\title{
A vocabulary of physical and rehabilitation medicine in French and Ukrainian - the comparative analysis
}

\section{[Le vocabulaire de la medecine physique et de readaptation en francais et en ukrainien : l'analyse comparative]}

\author{
Roman Pomirko - Rostyslav Koval - Olga Romanchuk - Olesya Cherkhava \\ - Nataliia Bazyliak
}

DOI: 10.18355/XL.2019.12.01XL.09

\begin{abstract}
The article is devoted to the comparative analysis of terms of physical and rehabilitation medicine in French and Ukrainian. The material for the research has been selected from the most modern specialized glossaries and dictionaries. We have investigated denominations of professions; terms which are used to nominate the tests, scales and classifications; eponyms and toponyms; abbreviations; loan words; synonyms. Main models of formation of terms in the field have been revealed. Also it has been found out that we have three basic tendencies in French - active use of borrowed terms from English; a great number of different abbreviations; functioning of synonyms. We consider that Ukrainian terminology of physical and rehabilitation medicine is on the way of its unification and standardization. The main task is to compile the first French-Ukrainian dictionary of physical and rehabilitation medicine which will contain a translation of denominations of occupations; most common defects, disorders and diseases; scales and tests; means and methods of rehabilitation; abbreviations.
\end{abstract}

Key words: term, vocabulary, analysis, physical and rehabilitation medicine, French, Ukrainian

\section{Résumé}

L'article est consacré à l'analyse comparative du vocabulaire de la médecine physique et de réadaptation / MPR en français et en ukrainien. Nous avons choisi le matériel pour notre recherche en utilisant les dictionnaires et les glossaires les plus actuels. Nous avons analysé les dénominations de professions; les tests, les échelles et les classifications; les éponymes et les toponymes; les sigles; les emprunts; les synonymes. On a établi les principaux modèles de formation de termes en question. En français de MPR nous avons les trois tendances majeures - l'utilisation active des emprunts anglais et des abréviations; le fonctionnement de la synonymie. Nous considérons que la terminologie ukrainienne de MPR est maintenant en voie de l'unification. La tâche urgente consiste en rédaction pour la première fois d'un dictionnaire français-ukrainien de MPR qui contiendra la traduction de termes concernant les professions; les défauts, les troubles et les maladies; les échelles et les tests; les moyens et les méthodes de MPR; les sigles.

Mots-clés: terme, vocabulaire, analyse, médecine physique et de réadaptation / MPR, français, ukrainien

\section{Introduction}

Selon l'Organisation Mondiale de la Santé, la réadaptation est un processus complexe qui a pour but de rétablir la valeur sociale de l'individu. De manière plus générale, il s'agit d'assurer le passage d'une prise en charge médicale à l'aide et l'accompagnement pour une vie sociale la mieux adaptée et la plus normale possible (Zribi, 2011: 281). La réadaptation constitue un domaine vaste et complexe qui XLinguae, Volume 12 Issue 1XL, January 2019, ISSN 1337-8384, eISSN 2453-711X 
embrasse toutes les facettes d'un être humain ayant des déficiences. Une grande quantité de mots sont nécessaires à sa description, mots qui doivent être choisis, définis et décrits (Blouin, Vallejo Echeverri, 2010).

En prenant en considération des hostilités qui se poursuivent dans l'est de l'Ukraine depuis 2014, les spécialistes nationaux ont un besoin urgent de réadaptation des soldats ukrainiens ainsi que des victimes d'un conflit militaire. Malheureusement, les guerres influencent directement sur l'évolution de cette branche de médecine. Les conséquences de la Seconde Guerre mondiale contribuent au développement de réadaptation à cause du nombre sans précédent de personnes handicapées qui doivent être traitées pour qu'ils puissent retourner à la vie normale dans la société.

Nous sommes d'accord avec Julia Facione-Roger qui dit qu'une nouvelle typologie de victime de guerre est née. La médecine militaire n'avait encore jamais eu à traiter des patients aux blessures si complexes. Elle doit ainsi relever de nouveaux défis en matière de rééducation, de réadapation, de reconversion et de suivi au long cours de ces patients (Facione-Roger, 2010: 47). De surcroît, il ne faut pas oublier les personnes handicapées de la naissance, à cause d'une maladie, des accidents du travail, suite aux catastrophes (comme Tchernobyl en 1986). Dans certains cas, la faute du médecin et son incompétence professionnelle peuvent entraîner un handicap. La vie quotidienne d'un handicapé change radicalement ainsi que le mode de vie de sa famille, ses amis et tous ceux qui entourent cette personne.

En Ukraine, il existe de nombreuses centres de la médecine physique et de réadaptation. Les spécialistes ukrainiens sont connus bien au-delà des frontières de pays. Par exemple, le professeur Volodymyr Kozyavkin qui a développé et mis en œuvre la pratique du système de réadaptation intensive neurophysiologique pour le traitement de diverses formes de paralysie cérébrale.

La médecine physique et de réadaptation joue un grand rôle dans le traitement des maladies, la restauration des fonctions perdues après les traumas et les blessures, la rééducation fonctionnelle et professionnelle, de même que dans la réduction du fardeau de l'invalidité des personnes infirmes. Elle augmente l'espérance de vie, elle s'utilise dans les buts thérapeutiques et prophylactiques dans le processus complexe de réadaptation. Les questions terminologiques de cette branche en Ukraine sont encore loin d'être résolues et appartiennent aux problèmes prioritaires (Hertsyk, 2016). Les relations entre un spécialiste de MPR et une personne handicapée qui maîtrisent soit le français, soit l'ukrainien nécessitent un dictionnaire spécialisé français-ukrainien et vice versa. Ajoutons qu'à ce jour aucun dictionnaire médical français-ukrainien n'est pas élaboré. Ce fait pose les problèmes pour la communication professionnelle entre les physiothérapeutes, les ergothérapeutes, les kinésithérapeutes, les rééducateures, les orthoprothésistes et d'autres spécialistes.

Envisageons les principaux dictionnaires français et ukrainiens de la médecine physique et de réadaptation. Notons que les questions qui concernent la terminologie de MPR en français, sont analysées dans les travaux de Maurice Blouin, Jacques Monet, Claude Hamonet, Jean-Michel Wirotius, en ukrainien - dans les articles d'Andriy Hertsyk, Rostyslav Koval et Oksana Tyravska.

En 1972, Herman Kamenetz et Georgette Kamenetz publient le "Dictionnaire de médecine physique, de rééducation et de réadaptation fonctionnelles". La portée du dictionnaire se limite aux fonctions motrices et comporte environ 2,500 termes (Blouin, Vallejo Echeverri, 2010). En 1981, le "Glossaire de la réadaptation profesionnelle et de l'emploi des handicapés" voit le jour. En 1986, Sophie Janik, Lise Brunet et Louis-Luc Lecompte publient le "Thésaurus: personne handicapée. Thésaurus à schémas fléchés de descripteurs pour l'analyse et la recherche des documents et du matériel avec leurs équivalents en langue anglaise". À Montpellier (France), le travail "Lexique et manuel Anglais-Français pour la Rééducation et la Réadaptation" sort en 1990. Le dictionnaire propose la traduction de termes concernant l'anatomie, l'appareillage, l'ergothérapie, l'évaluation du handicap, la 
kinésithérapie et le sport. En 1995, Maurice Blouin écrit le "Dictionnaire de réadaptation - termes techniques d'évaluation, Tome 1", qui traite les aspects physiques, psychologiques, sociaux et envitonnementaux de la réadaptation. Il comprend l'index anglais-français et français-anglais avec 924 termes. En 1997, Maurice Blouin présente le "Dictionnaire de la réadaptation. Tome 2. Termes d'intervention et d'aides techniques" avec l'index français-anglais et anglais-français, qui comporte 1153 termes (Blouin, Vallejo Echeverri, 2010).

Un peu plus tard, en France, Michel Dufour et Michel Gedda publient le "Dictionnaire de kinésithérapie et réadaptation", qui regroupe et définit les termes techniques et scientifiques issus de la pratique et de la culture de la masso-kinésithérapie francophone. En plus, il comporte les termes les plus usités de domaine connexes à la kinésithérapie tels que l'anatomie, la biomécanique, la physiologie, la méthodologie etc. Ces termes sont définis selon une approche kinésithérapeutique. En ce qui concerne la présentation, les entrées sont classées par ordre alphabétique et présentées selon un modèle homogène intégrant (Dufour, Gedda, 2007). Le "Dictionnaire du handicap" ( $7^{\mathrm{e}}$ édition) de Gérard Zribi et Dominique Poupée-Fontaine voit le jour en 2011, proposant une grande variété de termes autour du handicap. Au total, 400 articles, précédes d'une brève chronologie de la politique du handicap, suivis d'un index de près de 500 entrées et d'un guide pratique destiné aux usagers et à leurs familles (Zribi, Poupée-Fontaine, 2011).

Les spécialistes ukrainiens accordent aussi un vif intérêt pour la terminologie de MPR dans leurs travaux. Nous citons, entre autres, "Les concepts de base et les termes de l'éducation physique, de remise en forme et de réadaptation" (1998); "La terminologie médicale, biologique et de réadaptation pour les étudiants des spécialités Réadaptation physique, Culture physique" (2002); le "Dictionnaire des termes et des expressions de réadaptation physique" (2002), qui est publié à Lviv et contient 700 termes et expressions qui définissent les principaux groupes de concepts : les processus et les structures morphologiques du corps humain ; les noms, les formes de déroulement et les signes de diverses maladies (les symptômes et les syndromes) ; les facteurs environnementaux qui ont un impact positif ou négatif sur le corps humain ; les méthodes de diagnostic, de prévention et de traitement des maladies ; les domaines et les branches des sciences médicales, biomédicales, psychologiques et pédagogiques ; les principes et les formes organisationnelles de prestations des services médicaux, préventifs et de réadaptation aux handicapés ; les appareils, les instruments, les outils et autres dispositifs techniques à des fins de rééducation. Pour presque chaque terme, les auteurs donnent l'étymologie et une brève définition. Un peu plus tard, les travaux suivants sont publiés : "Le dictionnaire encyclopédique des termes, utilisés en physiothérapie (médecine physique)" (2004, Kyïv; en russe, mais avec le vocabulaire russe-ukrainien des termes) ; "La réadaptation médicale : les standards contemporains, les tests, les échelles et les critères de performance. La physiothérapie par résonance de faible intensité et son application en médecine de réadaptation" (2007) ; "La terminologie de la spécialité Santé de l'homme" (2009) ; "Les principes fondamentaux de recherches diagnostiques en réadaptation physique" (2010) ; "Le glossaire pour les étudiants de la spécialité Santé de l'homme (Réadaptation physique)" (2010) ; "Les concepts de base et les termes de maintien de la santé et de réadaptation physique dans le système de l'éducation" (2011), présentant l'interprétation des concepts de base utilisés dans le cycle d'apprentissage des disciplines sportives, médicales, biologiques et pédagogiques. Il convient de nommer aussi le dictionnaire "La réadaptation physique. La gymnastique médicale. La kinésithérapie" en russe (Moscou, 2010), avec les concepts concernant la gymnastique médicale, le massage, la physiothérapie et la chiropractie. Ce sont les dictionnaires raisonnés unilingues.

XLinguae, Volume 12 Issue 1XL, January 2019, ISSN 1337-8384, eISSN 2453-711X 


\section{Le but de recherche}

Le but de notre recherche consiste en analyse comparative de la terminologie de MPR en français et en ukrainien. Les termes sont tirés des dictionnaires mentionnés cidessus. Le besoin d'études de particularités lexicales et sémantiques en prenant en considération le niveau de développement d'outils et de méthodes de MPR en France et au Canada, le travail lexicographique et l'expansion rapide de cette science en Ukraine, la coopération des scientifiques et des conférences internationales, soulignent la nécessité de l'analyse des termes de MPR.

\section{Méthodologie}

Pour atteindre les objectifs fixés, on a utilisé les méthodes suivantes: l'échantillonnage (pour composer le registre des termes), l'analyse de formation de termes (pour l'étude des particularités lexicales et sémantiques), la méthode descriptive (pour l'interprétation des concepts), la méthode comparative (afin de comparer les termes en français et en ukrainien). La portée scientifique de la recherche consiste en première tentative de rédiger un dictionnaire français-ukrainien de MPR.

\section{Les résultats}

Avant de présenter les résultats de notre analyse, nous nous posons une question quels termes appartiennent au domaine de MPR ? Par exemple, dans le "Dictionnaire de kinésithérapie et réadaptation" les auteurs écrivent que dans le domaine de la terminologie, décider de recenser les mots d'un champ professionnel et de leur attribuer un sens ne peut être qu'une aventure. Il s'agit d'explorer les connaissances heuristiques et pratiques, dans une vision la plus vaste possible, afin d'identifier des mots et concepts utilisés à un moment donné (Dufour, Gedda, 2007). Dans le "Dictionnaire du handicap" on trouve les définitions et classifications des handicaps et des maladies qui les causent; les principaux éléments de politique sociale; les caractéristiques des prestations, établissements et services spécialisés, ainsi que les droits des usagers; les professionnels œuvrant auprès des personnes handicapées; les courants de pensée, méthodes éducatives, approches thérapeutiques et pratiques sociales (Zribi, Poupée-Fontaine, 2011). Donc, la sélection des termes pour l'analyse est subjective et dépend, tout d'abord, des connaissances et des compétences professionnelles du chercheur.

On appelle le terme de MPR un mot ou une expression désignant les concepts professionnels de cette industrie, dont le contenu se reflète dans la définition, et qui fait partie intégrante de la terminologie en question. Il doit être mise en œuvre et codifié, précis et concis, indépendant du contexte et conforme aux exigences de la langue littéraire. La terminologie de MPR en français et en ukrainien se développe rapidement, ce qu'on explique à la fois par des facteurs linguistiques et extralinguistiques, y compris l'introduction de nouveaux outils pour le diagnostic, le traitement et la réadaptation, le développement des prothèses sophistiquées etc.

Nous avons analysé les dénominations de professions de MPR; les termes pour nommer les échelles et les classifications; les éponymes et les toponymes; les sigles, les abréviations et les acronymes; les emprunts et les synonymes de MPR en français et en ukrainien.

\section{La formation de termes pour dénommer les professions de MPR}

La médecine physique et réhabilitation est une spécialité à part entière de la médecine, marquée par une forte pluridisciplinarité et des interconnexions professionnelles à divers niveaux. Le spécialiste en réadaptation a la charge du diagnostic, de la prévention, du traitement et du suivi de la réadaptation de patients de tout âge, atteints d'affections invalidantes et porteurs de comorbidités (Kramers-de Quervain, Knüsel, 2012). Aucune profession ne peut prétendre réunir toutes les connaissances nécessaires pour réaliser complètement et efficacement les évaluations, les 
interventions et la gestion de la réadaptation. C'est pourquoi une pratique multidisciplinaire regroupant des professionnels dotés d'un pouvoir d'évaluation et d'intervention égal est essentielle à une réadaptation intégrale (Blouin, Vallejo Echeverri, 2010). Notons qu'il existe aujourd'hui en Ukraine le besoin en experts qui ont suffisamment de connaissances pour mener des enquêtes indépendantes, faire un choix entre les techniques de réadaptation, ayant un stock suffisant de compétences pratiques et qui seraient capables de préparer son patient à vivre dans les nouvelles conditions en situation de handicap (Prystupa, Vovkanych, 2011).

Donc, pour nommer une profession de MPR en français on utilise le plus souvent le suffixe -iste: épithésiste $(\mathrm{m}, \mathrm{f})$; nutritionniste $(\mathrm{m}, \mathrm{f})$; oculariste $(\mathrm{m}, \mathrm{f})$; orthésiste $(\mathrm{m}, \mathrm{f})$; orthopédiste $(\mathrm{m}, \mathrm{f})$; orthophoniste $(\mathrm{m}, \mathrm{f})$; orthoptiste $(\mathrm{m}, \mathrm{f})$; prothésiste $(\mathrm{m}, \mathrm{f})$; le suffixe -ien: chirurgien, -ne $(\mathrm{m}, \mathrm{f})$; clinicien, -ne $(\mathrm{m}, \mathrm{f})$; technicien, -ne (m, f); le suffixe -eur: appareilleur $(\mathrm{m})$; masseur, -se $(\mathrm{m}, \mathrm{f})$; rééducateur, -trice $(\mathrm{m}, \mathrm{f})$; les suffixes -iatre et -ier: brancardier $(\mathrm{m})$; infirmier, -ère $(\mathrm{m}, \mathrm{f})$; physiatre $(\mathrm{m}, \mathrm{f})-\mathrm{le}$ terme est employé au Canada; podiatre $(\mathrm{m}, \mathrm{f})$ - le terme est employé au Canada; l'élément d'origine grecque -logue: audiologue (m, f); balnéologue (m, f); épileptologue $(\mathrm{m}, \mathrm{f})$; kinésiologue $(\mathrm{m}, \mathrm{f})$; podologue $(\mathrm{m}, \mathrm{f})$; postérologue $(\mathrm{m}, \mathrm{f})$; récréologue $(\mathrm{m}, \mathrm{f})$; l'élément d'origine grecque -thérapeute: art-thérapeute $(\mathrm{m}, \mathrm{f})$; chimiothérapeute $(\mathrm{m}, \mathrm{f})$; ergothérapeute $(\mathrm{m}, \mathrm{f})$; hydrothérapeute $(\mathrm{m}, \mathrm{f})$; massothérapeute $(\mathrm{m}, \mathrm{f})$; musicothérapeute $(\mathrm{m}, \mathrm{f})$; physiothérapeute $(\mathrm{m}, \mathrm{f})$; radiothérapeute $(\mathrm{m}, \mathrm{f})$; vertébrothérapeute $(\mathrm{m}, \mathrm{f})$. Nous observons la synonymie de termes formés avec -iste / -logue: neurologiste (m, f) / neurologue (m, f); physiologiste $(\mathrm{m}, \mathrm{f}) /$ physiologue $(\mathrm{m}, \mathrm{f})$; radiologiste $(\mathrm{m}, \mathrm{f}) / \operatorname{radiologue}(\mathrm{m}, \mathrm{f})$ / radiographe $(\mathrm{m}, \mathrm{f})$; rhumatologiste $(\mathrm{m}, \mathrm{f})$ / rhumatologue $(\mathrm{m}, \mathrm{f})$; traumatologiste $(\mathrm{m}, \mathrm{f})$ / traumatologue (m, f). On observe également la synonymie de termes formés avec les différents thèmes: oncologue $(m, f) /$ cancérologue $(m, f)$; otologiste $(m, f) /$ auriste $(\mathrm{m}, \mathrm{f})$; pneumologue $(\mathrm{m}, \mathrm{f}) /$ inhalothérapeute $(\mathrm{m}, \mathrm{f})$ (le terme est employé au Canada).

Le procédé de formation appelé la composition est aussi productif dans la terminologie de MPR: aide-soignante (f); assistant (m) de service social; assistantsenior $(\mathrm{m})$; chirurgien-orthopédiste $(\mathrm{m})$; conseiller $(\mathrm{m})$ d'orientation; éducateur $(\mathrm{m})$ physique; masseur-kinésithérapeute $(\mathrm{m}, \mathrm{f})$; massothérapeute $(\mathrm{m}, \mathrm{f})$; moniteur $(\mathrm{m})$ en réadaptation; orthopédagogue $(\mathrm{m})$; orthésiste-prothésiste $(\mathrm{m})$; ostéopraticien $(\mathrm{m})$; psychomotricien $(\mathrm{m})$; pédagogue $(\mathrm{m})$ curatif; spécialiste $(\mathrm{m})$ des déficiences; travailleur $(\mathrm{m})$ social. On utilise très souvent un mot médecin $(\mathrm{m})$ : médecin $(\mathrm{m})$ climatothérapeute; médecin $(\mathrm{m})$ de médecine physique et de réadaptation; médecin (m) de rééducation fonctionnelle; médecin $(\mathrm{m})$ de santé publique; médecin $(\mathrm{m}) \mathrm{du}$ sport; médecin $(\mathrm{m})$ thermal.

En ukrainien, le plus productif est l'élément d'origine grecque -лог: аудіолог; бальнеолог; дефектолог; дієтолог; епілептолог; імунолог; кардіолог; кінезіолог; комбустіолог; курортолог; мамолог; нарколог / токсиколог; невролог; онколог; отолог; подолог; психолог; пульмонолог; реабілітолог; ревматолог; рекреолог; сурдолог; травматолог. On utilise également les suffixes -ucm et -icm: гігієніст; масажист; нутріціоніст; ортофоніст / логопед; протезист; le suffixe -amp: подіатр; психіатр; фтизіатр; l'élément d'origine grecque -mерапевm: вертебротерапевт; гідротерапевт; ерготерапевт; кліматотерапевт; психотерапевт; фізіотерапевт; хіміотерапевт. La composition est utilisée souvent: анестезіологреаніматолог; аудіопротезист; мануальний терапевт / хіропрактик; масажист і методист з лікувальної фізичної культури; ортопедагог; працівник соціальної служби; сурдопедагог; тифлопедагог; хірург-ортопед.

\section{La formation de termes pour dénommer les tests et les échelles en MPR}


Les tests et les échelles jouent un rôle clé dans l'évaluation des violations de la vie quotidienne des patients et des handicapés. Ces tests doivent posséder les qualités suivantes: la fiabilité, la validité et la sensibilité. Dans notre recherche nous divisons les termes en trois groupes: 1) test (m) ou épreuve (f); 2) échelle (f) / score (m) / grille (f) / batterie (f) / questionnaire (m); 3) classification (f) / index (m) / indice (m). Le premier groupe "test" (m) / "épreuve" (f) - "тест" contient les emprunts anglais FAT / Frenchay arm test (échelle d'évaluation fonctionnelle de la main dans le cadre d'une hémiplégie) - тест для руки Френчай; belly press-test (épreuve testant l'intégrité du muscle subscapulaire) - тест на цілісніть підлопаткового м'язу; padtest (épreuve non invasive de quantification de l'incontinence urinaire qui consiste à peser une protection hygiénique avant et après un laps de temps, plus ou moins occupé d'effort physique, de façon à en déduire la quantité d'urine perdue) / test (m) de la garniture - неінвазивний тест вимірювання ступеня нетримання сечі. On observe l'utilisation parallèle d'un terme français, d'un emprunt anglais et d'un éponyme: Depoorter's test / knee to shoulder test / sacroiliac rocking test / signe (m) de la flexion croisée - тест на цілісність крижово-клубової і крижово-горбової зв'язок; midline sacral thrust / prone springing test / sacral apex pressure test / sacral base springing test / sacral pressure test / sacral thrust test / test (m) de Coste et Illouz / pression (f) sur la crête sacrée - тест на визначення ураження передніх крижовоклубових зв'язок, задньої крижово-клубової зв'язки, передньої частини суглобової капсули крижово-клубового та попереково-крижового суглобів. Les éponymes sont utilisés fréquemment: test $(\mathrm{m})$ de Babinski-Anton (épreuve clinique destinée à tester l'asynergie cérébelleuse) - тест Бабінскі-Антона; test (m) de Fukuda (épreuve mettant en évidence une éventuelle asymétrie tonique posturale) - тест Фукуди; test (m) de Gerber (épreuve active testant l'integrité du subscapulaire) - тест Жербера; test (m) de Godfrey (épreuve testant l'integrité du ligament croisé postérieur du genou) - тест на цілісність задньої схрещеної зв'язки коліна; test (m) de Kendal (épreuve évaluant la force des muscles abdominaux) - тест для оцінки сили черевних м'язів; test $(\mathrm{m})$ de Sollerman (épreuve fonctionnelle destinée à évaluer les préhensions) - тест Соллермана; test (m) de Risser (technique d'évaluation radiographique de la maturité osseuse, à partir de l'ossification du noyau de la crête iliaque visualisée sur un cliché de face) - тест Piccepa. Il est à noter que dans certains cas le nom du test est clair et on comprend bien sa destination: test (m) d'aptitude - тест на виявлення здібностей; test (m) de continence urinaire - тест на тримання сечі. Cependant, seulement les professionnels peuvent connaître le but d'un test $(\mathrm{m})$ à la sueur (le dosage du chlore dans la sueur du patient atteint de la mucoviscidose) - тест на вміст хлориду натрію в потовій рідині (при муковісцидозі). On peut traduire le terme français "manœuvre" (f) en ukrainien comme "тест": manœuvre (f) de Cabot (épreuve destinée à mettre en évidence une souffrance du ménisque latéral du genou) - тест для визначення болю бокового меніску коліна; manœuvre (f) de Finkelstein / signe (m) du soutien-gorge (épreuve testant l'intégrité du long abducteur du pouce, le plus souvent dans le cadre de ténosynovite) - тест на цілісність довгого відвідного м'язу великого пальця руки; manœuvre (f) de Léri / signe $(\mathrm{m})$ de Lasègue inversé (épreuve destinée à détecter l'irritation des racines crurales par une hernie discale, par compression de la protrusion discale sur la racine concernée) - тест для визначення подразнення стегнового нерва внаслідок диско-радикулярного конфлікту; manœuvre (f) / déclic (m) de Mac Murray (épreuve testant l'intégrité des ménisques du genou, notamment de la corne postérieure) - тест для виявлення ушкодження меніска колінного суглобу. On observe les cas des éponymes avec l'utilisation d'un terme "épreuve": épreuve (f) de Martinet (épreuve fonctionnelle d'effort) - функціональний тест на зусилля (навантажувальний тест) Мартіне; épreuve (f) de Mingazzini (épreuve proposée pour détecter un déficit moteur fruste d'origine centrale) - проба Мінгаззіні (тест для діагностики легкого парезу кінцівок); épreuve (f) de Möberg (épreuve clinique 
d'évaluation fonctionnelle de la sensibilité de la main) - клінічний тест для функціональної оцінки чутливості руки; épreuve (f) de Stewart-Holmes (épreuve destinée à détecter une asynergie musculaire utilisée, notamment, dans le cadre du syndrome cérébelleux) - тест на виявлення м'язевої асинергії (порушення координації) при мозочковому синдромі.

Le deuxième groupe "échelle" (f) / "score" (m) / "grille" (f) / "batterie" (f) - "шкала" contient les termes formés avec "échelle" (f): échelle (f) de gravité - шкала важкості; échelle (f) d'Orgogozo (échelle d'évaluation de l'état neurologique d'un sujet souffrant d'un infarctus sylvien) - шкала оцінки неврологічного стану людини з інсультом середньої мозкової артерії; échelle (f) de capacités motrices du tétraplégique шкала рухових можливостей пацієнта $з$ тетраплегією; échelle (f) d'acuité - шкала визначення гостроти зору; échelle (f) de douleur neuropathique / questionnaire (m) DN 4 - шкала оцінки невропатичного болю; échelle (f) de Klein-Bell (échelle d'évaluation des activités de la vie quotidienne) - шкала оцінки активностей щоденного життя; échelle (f) de Rankin (échelle d'évaluation de l'invalidité d'un sujet victime d'accident vasculaire cérébral) - шкала Ренкіна (оцінка функціональних можливостей хворих після інсульту); échelle (f) de risque de Norton (échelle d'évaluation du risque de développer une escarre, basée sur 5 critères: condition physique générale, état mental, activités quotidiennes, mobilité, incontinence) - шкала оцінки ризику розвитку відлежин. Les termes "cotation" (f) et "guide" (m) dans certains cas on traduit en ukrainien comme "шкала": cotation (f) de Merle d'Aubigné (échelle d'évaluation du retentissement fonctionnel de la coxarthrose) - шкала Мерля Добіньї (оцінка функціонального впливу коксартрозу); GEVA / guide (m) d'évaluation des besoins de compensation des personnes handicapées - шкала оцінки компенсаторних потреб людей 3 обмеженими фізичними можливостями. Il у a des emprunts anglais: PSFS / Penn spasm frequency scale (échelle analytique d'auto-évaluation de la fréquence de spasmes liés à une spasticité importante d'origine médullaire) - аналітична шкала самооцінки частоти спазмів, пов'язаних зі значною спастичністю спинномозкового походження; les termes formés avec "score" (m) - “шкала": score (m) de Beighton (échelle d'évaluation de l'hypermobilité articulaire) - шкала Бейтона (оцінка надмірної рухливості суглобів); score (m) de Constant / d'évaluation scapulaire (échelle d'évaluation analytique et fonctionnelle de l'épaule pour des sujets souffrant de la coiffe des rotateurs) - шкала аналітичної i функціональної оцінки плеча у людей з пошкодженням ротаторної манжети; les termes formés avec "batterie" (f) - “шкала": batterie (f) rapide d'évaluation frontale / BREF / en anglais Frontal Assessment Battery / FAB - шкала оцінки синдрому лобної долі / лобний (нейропсихологічний) синдром (закономірне поєднання симптомів, спричинене масивним, переважно двостороннім ураженням лобних долей головного мозку); les termes formés avec "grille" (f) - "шкала": AGGIR / grille (f) autonomie (f) gérontologique - groupe (m) iso-ressource (échelle d'estimation du degré d'autonomie d'un sujet âgé afin de le classer en six groupes isoressources, même mobilisation de ressources pour faire face à la dépendance, déterminant le besoin d'assistance pour les actes de la vie quotidienne en France) шкала для оцінки рівня самостійності або втрати самостійності людей (здебільшого похилого віку; використовується у Франції). Ce groupe contient également "questionnaire" (m) / “опитувач" / en anglais "questionnaire". Nous avons les emprunts anglais: GHQ / General health questionnaire (concerne la santé) опитувач загального здоров'я; LHS / London Handicap Scale (concerne la qualité de vie quotidienne) - опитувач якості щоденного життя; SGRQ / Saint-Georges respiratory questionnaire (concerne les patients avec l'obstruction de voies respiratoires) - опитувач госпіталю Святого Георгія (призначений для визначення стану здоров'я, рівня повсякденного життя та благополуччя у 
пацієнтів з обструктивними захворюваннями дихальних шляхів); ODI / Oswestry Disability Index / the Oswestry Low Back Pain Disability Questionnaire (concerne le lumbago) - шкала оцінки ступеню порушення життєдіяльності, що зумовлене болями у попереку (патологією хребта). Enfin, le dernier groupe "classification" (f) - “класифікація" contient: classification (f) de Luria (l'aphasie) - класифікація афазії Лурія; classification (f) de Child (sévérité de la cirrhose) - класифікація Чайлда (цироз печінки); classification (f) internationale des maladies et des causes de décès - міжнародна класифікація хворіб та причин летальних випадків; classification (f) TNM de la tumeur - класифікація пухлин по системі TNM (пухлина-лімфовузли-метастази). En MPR on utilise souvent les indices (on traduit ce terme en ukrainien comme "оцінка", "шкала", "показник"): indice (m) de dissociation des seuils / test (m) de Fishgold (épreuve d'électrodiagnostic proposée dans le cadre du traitement de la paralysie faciale, pour évaluer la gravité d'une lésion neurogène périphérique et en suivre l'évolution) - оцінка важкості нейрогенного патологічного ураження; indice (m) de Katz (échelle d'évaluation de l'autonomie physique de la personne âgée souffrant d'affections chroniques évaluant sa capacité à réaliser six activités de la vie quotidienne) - індекс активностей повсякденного життя Катц; indice (m) fonctionnel de Duruöz (échelle quantifiant les foctions manuelles d'un sujet) - шкала для визначення рівня функціонального стану руки; indice $(\mathrm{m})$ fonctionnel de Lee (échelle de mesure de l'incapacité fonctionnelle produite par la polyarthrite rhumatoïde) - шкала вимірювання функціональної нездатності через ревматоїдний поліартрит; index (m) de Barthel (échelle d'évaluation de l'indépendance fonctionnelle et situationnelle du sujet âgé) - індекс активностей повсякденного життя Бартела.

\section{Les éponymes et les toponymes en MPR}

Après avoir analysé les dénominations des tests et des échelles en MPR, nous voyons que les éponymes sont très fréquents dans la terminologie en question. On emploie les éponymes pour: 1) nommer les troubles et les maladies : maladie (m) de Marfan I синдром Марфана I, du nom du pédiatre français Bernard-Jean Antonin Marfan (1858-1942) ; maladie (f) de Duchenne-Erb - параліч проксимальних відділів руки Дюшена-Ерба, du nom du médecin français Guillaume Duchenne (1806-1875) et du nom du neuropathologue allemand Wilhelm-Heinrich Erb (1840-1921) ; syndrome (m) de Down - синдром Дауна, du nom du médecin anglais John Langdon Haydon Down (1828-1896) ; maladie (f) de Parkinson - хвороба Паркінсона, du nom du médecin anglais James Parkinson (1755-1824) ; maladie (f) de Little - хвороба Літтла, du nom du médecin anglais William John Little (1810-1894) ; maladie (f) d'Alzheimer - хвороба Альцгеймера, du nom du médecin allemand Alois Alzheimer (1864-1915) ; fracture (f) de Dupuytren - перелом Дюпуйтрена, du nom du chirurgien français Guillaume Dupuytren (1777-1835). 2) pour nommer les appareils de MPR : harnais (m) de Pavlik (orthèse d'abducion et de flexion des hanches utilisée pour le traitement orthopédique de la maladie luxante des hanches du nourrisson au stade de la hanche luxable) - стремено Павліка (призначене для лікування дисплазії, вроджених вивихів, інших дефектів кульшових суглобів у дітей), du nom du pédiatre tchèque Arnold Pavlik (1902-1965); attelle (f) de Brown (orthèse de correction du pied-bot constituée de deux plaquettes articulées entre elles pour corriger le pied-bot dans les trois plans de l'espace) - шина Брауна, du nom du chirurgien anglais né en Australie Denis John Brown (1892-1967); brosses (f, pl) de Garros-Demol (batterie d'instruments pour la rééducation de la sensibilité superficielle des doigts) - щітки Гарро-Демоль, du nom du kinésithérapeute français Jean-Claude Garros (1943-2003) et du technicien français Willy Demol (contemporain) ; cadre (m) de Guttmann (appareil de marche pour faciliter la déambulation en trois temps du blessé médullaire) - ходунці (рама) Гутмана, du nom du médecin brittanique d'origine allemande Ludwig Guttmann (1899-1980) ; orthèse (f) de Perlstein 
(mécanisme de jonction réglable permettant de paramétrer le débattement articulaire entre le montant latéral d'une orthèse jambière et la chaussure) - ортез Перлштайна, du nom du médecin nord-américain Meyer Aaron Perlstein (1902-1969) ; orthèse (f) de Nancy-Hylton (orthèse suro-pédieuse dynamique utilisée en pédiatrie pour réduire et/ou compenser les perturbations posturales de la cheville lors des activités de la vie quotidienne) - ортез Ненсі-Хілтон, du nom de la kinésithérapeute nord-américaine Nancy M. Hilton (contemporaine). 3) pour nommer les méthodes de MPR : méthode (f) de Ajuriaguerra (méthode de rééducation psychotonique basée sur le déconditionnement et l'écoute du corps par le dialogue tonico-émotionnel) - метод Ажюріягерри, du nom du neuropsychiatre et psychanalyste français d'origine espagnole Julian de Ajuriaguerra (1911-1993) ; méthode (f) de Rocher (méthode de renforcement musculaire dynamique) - метод Роше, du nom du chirurgien orthopédiste français Christian Rocher (1908-2002); manœuvre (f) de Cyriax (manœuvre antalgique, introduite en France par Troisier en 1951, qui consiste en une friction particulière, appelée aussi massage transversal profond ou frotte, ponçage, sur une lésion ligamentaire ou tendineuse) - прийом Сайрієкса, du nom du médecin orthopédiste anglais James Henry Cyriax (1904-1985). De temps en temps nous observons l'utilisation seulement du nom propre: Klapp (méthode de rééducation quadrupédique des déviations modérées de la colonne vertébrale et d'autres affections thoraco-vertébrales) - метод Кляппа, du nom de l'orthopédiste allemand Rudolf Klapp (1873-1949). 4) le quatrième groupe contient les nominations de classifications, des indices, des échelles et des tests: indice $(\mathrm{m}) /$ coefficient $(\mathrm{m}) \mathrm{de}$ Tiffeneau (rapport du volume expiratoire maximal en une seconde sur la capacité vitale permettant d'indiquer une obstruction bronchique) - індекс / коефіцієнт Тіффно (відношення форсованого видиху за першу секунду до життєвої ємності легень), du nom du physiologiste français Robert Tiffeneau (1910-1961) ; score (m) de Laskin (échelle d'évaluation analytique et fonctionnelle d'une prothèse de genou posée sur un terrain rhumatismal) - шкала Ласкіна (шкала аналітичної та функціональної оцінки протеза коліна, який ставиться на ушкоджену ревматизмом ділянку), du nom du chirurgien orthopédiste nord-américan Richard S. Laskin (contemporain); échelle (f) de Kurtzke (échelle de gravité de la sclérose en plaques) - шкала Куртцке (оцінка важкості розсіяного склерозу), du nom du neurologue nord-américain John F. Kurtzke (contemporain); épreuve (f) de Garcin (épreuve destinée à mettre en évidence un déficit moteur fruste du membre supérieur) - тест Гарсена, du nom du neurologue français Raymond Garcin (1897-1971); échelle (f) d'Ashworth (cotation analytique de la spasticité dans le cadre de la sclérose en plaques) - шкала Ашворта, du nom du neurologue anglais Bryan Ashworth (contemporain); indice (m) algofonctionnel de Lequesne (échelle d'évaluation du retenstissement de la coxarthrose et de la gonarthrose dans la vie du sujet) - індекс Лекесна (оцінка впливу коксартрозу і гонартрозу на життя пацієнта), du nom du rhumatologue français Michel Lequesne (contemporain); épreuve (f) de Barré (épreuve destinée à mettre en évidence un déficit moteur fruste de membres) - проба Барре (тест на виявлення слабкості кінцівок), du nom du neurologue français Jean Alexandre Barré (1880-1967). 5) les éponymes dérivés des personnages littéraires ou mythiques: syndrome (m) de Münchausen / pathomimie (f) / trouble (f) factice синдром Мюнхгаузена; syndrome (m) "Alice au pays des merveilles" / de Todd синдром Аліси в країні чудес.

Dans la terminologie de MPR on utilise également les toponymes: espalier (m) suédois - шведська стінка; gymnastique (f) suédoise - шведська гімнастика; massage (m) suédois - шведський масаж; massage (m) thaïlandais - тайський масаж; douche (f) écossaise / контрастний душ. Ils sont souvent utilisés pour dénommer les corsets: corset $(\mathrm{m})$ de Milwaukee - корсет Мілуокі (du nom de la ville de Milwaukee dans le Wisconsin, aux États-Unis, où le corset a été conçu en 1946 par

XLinguae, Volume 12 Issue 1XL, January 2019, ISSN 1337-8384, eISSN 2453-711X 
le professeur W. P. Blount); corset (m) de Boston - бостонський корсет (du nom de l'hôpital pour les enfants à Boston, aux États-Unis); corset (m) lyonnais - ліонський корсет (de la ville de Lyon en France, où ce corset a été conçu par Stagnara); corset (m) berckois - беркський корсет (de la ville Berck-sur-Mer en France); les prothèses et les orthèses: bras $(\mathrm{m})$ de l'Utah - рука Юта (prothèse myoélectrique d'appareillage du moignon brachial - de l'Université de Utah de Salt Lake City aux États-Unis); orthèse (f) de Copenhagen - копенгагенський ортез (pour le traitement de la courbure du pied, le nom vient de la capitale du Danemark - Copenhague, où elle a été mise au point); écharpe (f) de Montréal - монреальска пов'язка (orthèse de soutient du membre supérieur - du nom de la ville de Montréal au Canada où l'écharpe a été mise au point); les échelles et les classifications: échelle (f) de coma Glasgow / score (m) de Glasgow / en anglais Glasgow Coma Scale / GCS (échelle d'évaluation de la vigilance dans le cadre d'un coma, basée sur 3 réponses motrices de base) - шкала коми Глазго, de la ville de Glasgow en Écosse; test (m) de Vancouver (test d'évaluation d'une cicatrice) - ванкуверський тест (для оцінки важкості шрамів), de la ville de Vancouver au Canada où ce test a été mis au point; douleur (f) du rachis: auto-questionnaire de Dallas / DRAD / en anglais Dallas Pain Questionnaire / DPQ (échelle de la qualité de vie des sujets lombalgiques chroniques) - шкала оцінки якості життя людей, які страждають на болі в попереку, de la ville de Dallas aux États-Unis où ce questionnaire a été élaboré; douleur (f) enfant (m, f) San-Salvadour / DESS (échelle d'hétéro-évaluation comportementale de l'enfant polyhandicapé, qui ne communique pas ou difficilement) - шкала Сан-Сальвадур (шкала оцінки поведінки дитини з вадами), du nom de l'hôpital San-Salvadour de Hyères (France); échelle (f) de dorso-lombalgie de Québec / en anglais The Quebec Back Pain Disability Scale - шкала самооцінки впливу болей у грудному та поперековому відділах хребта на щоденне життя. Les toponymes sont utilisés pour dénommer les maladies et les syndromes: fièvre (f) du Colorado - гарячка Колорадо (de l'état du Colorado aux États-Unis); syndrome (m) de Jérusalem - єрусалимський синдром (de la ville de Jérusalem, en Israël); maladie (f) de Bornholm - хвороба Борнхольма (du nom de l'île de Bornholm dans la mer Baltique, qui appartient au Danemark).

\section{Les sigles, les abréviations et les acronymes en MPR}

En français de MPR on emploie très souvent les sigles, les abréviations et les acronymes. Pour les noms des organisations et des institutions nous avons: CPSS / Caisse (f) primaire de la sécurité sociale - первинна каса соціального страхування; AFIGAP / Association (f) Francophone Internationale des Groupes d'Animation de la paraplégie - Міжнародна франкомовна асоціація людей, уражених параплегією; ANPAA / Association (f) Nationale de Prévention en Alcoologie et Addictologie Національна асоціація попередження алкоголізму та залежності; APF / Association (f) des Paralysés de France - Асоціація паралізованих людей Франції; ANFE / Association (f) Nationale Française des Ergothérapeutes - Національна французька асоціація ерготерапевтів; SORNEST - Société (f) Régionale de Réadaptation du Nord-Est (en France) - Регіональне товариство реабілітації Півночі і Сходу (у Франціï). Pour nommer les professions nous avons: MK / masseur-kinésithérapeute (m) - масажист і фахівець 3 лікувальної фізичної культури; MCMK / moniteur-cadre (m) de masso-kinésithérapie - інструктор 3 кінезітерапії; MPR / médecin (m) physique et réadaptation - фахівець з фізичної та реабілітаційної медицини; AMM / auxiliaire (m) médical masseur - допоміжний медичний персонал (масажист); AMK / auxiliaire (m) médical kinésithérapeute допоміжний медичний персонал (кінезітерапевт) ; AVS / auxiliaire (m) de vie scolaire - помічник неповносправного учня; ASQ / aide-soignante (f) qualifiée кваліфікована доглядальниця. Pour dénommer les troubles et les maladies nous avons: IM / infirmité (f) motrice - рухова недостатність ; ALD / affection (f) de 
longue durée - довготривала втрата працездатності; ILMI / inégalité (f) de longueur des membres inférieurs - нерівна (різна) довжина нижніх кінцівок; NCB / névralgie (f) cervico-brachiale - шийно-плечова невралгія; RGO / reflux (m) gastroœsophagien - гастроезофагеальний рефлюкс; IRA / insuffisance (f) respiratoire aiguë - гостра дихальна недостатність; SEC / syndrome (m) d'épuisement chronique - синдром хронічного виснаження; TDR / trouble (m) du rythme cardiaque - порушення серцевого ритму; SEP / sclérose (f) en plaques - розсіяний склероз. Pour dénommer les échelles et les classifications nous avons: CIF / Classification (f) Internationale du Fonctionnement, du handicap et de la santé МКФ / Міжнародна класифікація функціонування, обмеження життєдіяльності та здоров'я; ECPA / échelle (f) comportementale d'évaluation de la douleur chez la personne âgée - шкала оцінки болю у людини похилого віку, яка повністю або частково не може спілкуватися. Pour dénommer les moyens de MPR nous avons: PTG / prothèse (f) totale de genou - повний протез коліна; PTH / prothèse (f) totale de hanche - повний протез тазостегнового суглобу; PTE / prothèse (f) totale d'épaule - повний протез плеча; FR / fauteuil (m) roulant - крісло-каталка; FRE / fauteuil (m) roulant électrique - крісло колісне з електричним приводом. Il y a les emprunts d'anglais: FRC / functional residual capacity - функціональна залишкова ємність легень; PASS / postural assessment scale for stroke patients - шкала оцінки постуральних можливостей у людини після порушення мозкового кровообігу; $\mathrm{HAD}(\mathrm{S})$ / Hospital Anxiety and Depression Scale - госпітальна шкала тривоги i депресіï. Parmi les sigles il y a les homonymes: CMP / Centre (m) de Médecine Préventive - Центр превентивної медицини et CMP / centre (m) médicopsychologique - медично-психологічний центр; DLM / dose (f) létale minimale мінімальна смертельна доза et DLM / drainage (m) lymphatique manuel лімфатичний ручний дренаж; $\mathrm{Fr}$ / fréquence respiratoire - частота дихання et FR / fauteuil (m) roulant - крісло колісне; FRE / fauteuil (m) roulant électrique - крісло колісне 3 електричним приводом et FRE / fréquence (f) respiratoire après effort частота дихання після навантаження; IR(C) / insuffisance (f) rénale (chronique) ниркова недостатність (хронічна) et IR(C) / insuffisance (f) respiratoire (chronique) - дихальна недостатність (хронічна). Les acronymes sont aussi fréquents: OLYMPE / orthèse (f) lyonnaise Massues à pression élastique - ліонський еластичний корсет Массю; EIFEL / échelle (f) d'incapacité fonctionnelle pour l'évaluation du lombalgique - шкала функціональних порушень для оцінки болю у попереку; OVALE / orthèse (f) vertébrale active lombaire d'élongation розтягуючий корсет для попереку; ANAP / Agence (f) nationale d'appui à la performance des établissements de santé et médico-sociaux - Національна агенція 3 питань вдосконалення роботи медичних та соціально-медичних закладів; MAIS / Mouvement (m) pour l'accompagnement et l'insertion sociale - рух з супроводу та соціальної інтеграції; SAMSAH / Services (m, pl) d'accompagnement médico-social pour adultes handicapés - послуги 3 медично-соціального супроводу дорослих неповносправних людей; SEGPA / sections (f, pl) d'enseignement général et professionnel adapté - секції з адаптованої загальної та професійної освіти; SESSAD / service $(\mathrm{m})$ d'éducation spéciale et de soins à domicile - служба 3 спеціальної освіти та догляду вдома. Il у а les acronymes empruntés d'anglais: RICE / rest, ice, compression, elevation - методика RICE (rest - спокій, ісе - лід, compression - стиснення, elevation - піднімання); PEDRO / physiotherapy evidence database - бібліографічна база даних результатів усіх проведених досліджень в рамках доказової медицини стосовно фізичної терапії. On emploie les sigles pour dénommer les certificats et les diplômes: CAFAD / certificat (m) d'aptitude aux fonctions d'aide à domicile - сертифікат, який дає право на надання допомоги неповносправному вдома; DEAVS / diplôme (m) d'État d'auxiliaire de vie sociale - диплом, що дає право супроводжувати людину 3 обмеженими 
можливостями, хворого або похилого віку у їхній повсякденній діяльності; CAFDES / certificat (m) d'aptitude à la fonction de directeur d'établissement social сертифікат на право виконання обов'язків директора соціального закладу; DDEEAS / diplôme $(\mathrm{m})$ de directeur d'établissement d'éducation adaptée et spécialisée - диплом директора закладу адаптованої та спеціалізованої освіти.

Il y a les sigles qui se composent de plusieurs composants: SYNERPA / Syndicat (m) national des établissements et résidences privés pour personnes âgées Національний синдикат закладів та приватних установ для людей похилого віку; COFEMER / Collège (m) Français des Enseignants universitaires de Médecine Physique et de Réadaptation - Французький Коледж для підготовки університетських викладачів галузі фізичної та реабілітаційної медицини; AGEFIPH / Association (f) de gestion du fonds pour l'insertion professionnelle des personnes handicapées - Асоціація керування коштами для професійного працевлаштування неповносправних людей; CTNERHI / Centre (m) Technique nationale d'études et de recherches sur les handicaps et les inadaptations - Технічний національний центр вивчення і дослідження фізичних вад і непристосованості; ASFORRED / Fédération (f) pour la Formation Continue des Associations (f, pl) de Médecins de Rééducation et de Réadaptation - Федерація з питань постійної освіти Асоціації лікарів - фахівців з реабілітації.

Les emprunts en MPR. Les termes empruntés en MPR sont utilisés pour désigner les défauts, les troubles et les maladies; les méthodes et les moyens de MPR; les tests, les échelles et les classifications. En français, pour dénommer les troubles et les pathologies nous avons les emprunts à l'anglais: freezing (blocage soudain, imprévisible et transitoire de la motricité volontaire observé dans le syndrome parkinsonien) / akinésie (f) paroxystique - пароксизмальна акінезія; burn-out / usure (f) des personnes - емоційне вигоряння; locked-in syndrom / LIS / syndrome (m) de verrouillage / coma (m) vigil - псевдокома; drop-attack (diminution soudaine du tonus postural avec dérobement des membres inférieurs et chute, sans perte de connaissance) - синкопальний вертебральний синдром; whiplash (brusque traumatisme de la région cervicale haute, consécutif à un aller-retour violent de la tête dans le plan sagittal, sur un cou non sécurisé) / coup (m) du lapin - травма спини, шиї, хребта від раптового різкого руху; tennis-elbow / tennis arm (enthésopathie de surcharge des muscles épicondyliens latéraux) / épicondylite (f) latérale - тенісний лікоть / запалення суглобової капсули ліктьового суглобу; golf-elbow (enthésopathie de surcharge des muscles épicondyliens médiaux, liée notamment à une flexion et inclinaison ulnaire excessives et répétées de la main lors de la prise du club de golf grip et à une tension musculaire exagérée et aux chocs répétés à chaque drive) / épicondylite (f) médiale - медіальний епікондиліт / лікоть гольфіста. Pour dénommer les moyens et les méthodes nous avons les emprunts à l'anglais: clapping (technique passive utilisée pour le décollement et l'évacuation des sécrétions bronchiques) - метод масажу, котрий використовується при ураженні органів дихання для кращого відходження мокротиння; cardio-training (ensemble des exercices physiques réalisés en centre de rééducation, salle de gymnastique, voire à domicile, et destinés à l'entraînement cardio-vasculaire, afin d'améliorer la fonction cardiaque) - сукупність фізичних вправ, спрямованих на покращення роботи серцево-судинної системи; computer-assisted motivating rehabilitation / CAMR (utilisation de jeux informatiques à piloter à l'aide d'un joystick ou d'un volant télécommandé pour inciter le patient à utiliser la main du côté hémiplégique) використання джойстика у відеоіграх 3 метою розроблення функціонування ураженої кінцівки. Il у a les emprunts à d'autres langues: terrainkur - теренкур / лікування дозованою ходьбою, à l'allemand; snoezelen (approche éducative faisant appel aux stimuli et aux sensations physiques des enfants et adultes handicapés mentaux graves ou polyhandicapés, voire aux déments) - снузелен / контрольована мультисенсорна стимуляція, du néérlandais. Pour nommer les appareils nous avons 
les emprunts à l'anglais: feeder (orthèse équilibrée de membre supérieur) - ортез для підтримки плеча; stepper (appareil reproduisant un effort de montée de marche utilisé pour le réentraînement à l'effort ou lors de sollicitation en chaîne fermée des muscles des membres inférieurs) - степер; standing-up (dispositif de posture en position debout utilisé chez les sujets ne pouvant maintenir cette position) / verticalisateur $(\mathrm{m})$ - вертикалізатор / стендер; medicin'ball (ballon lesté servant à travailler les lancers et réceptions avec une charge à des fins de musculation, de coordination ou d'équilibration) - медицинбол. Pour dénommer les échelles et les tests nous avons les emprunts d'anglais: shuttle walk test / SWT (épreuve fonctionnelle d'effort de course, proposée par Léger et Boucher en 1985 pour déterminer la vitesse maximale aérobie) / test (m) de la navette / course-navette (f) ступінчастий тест човникової ходьби “шатл-тест" (використовується для функціонального дослідження дихальної системи); Roland and Morris Disability questionnaire / échelle (f) d'auto-évaluation fonctionnelle d'un lombalgique - анкета Роланда-Морріса (вплив болю у попереку на порушення життєдіяльності); Rivermead motor assessment / RMA / échelle (f) d'évaluation des capacités motrices d'un sujet de moins de 65 ans victime d'accident vasculaire cérébral - оцінка рухових можливостей людей віком до 65 років після порушення мозкового кровообігу.

En ukrainien pour dénommer les troubles et les maladies nous avons les emprunts de français: коклюш - coqueluche (f); грип - grippe (f); тік - tic (m); рамол / фізично або розумово немічна людина - ramolli (m, f); кретинізм - crétinisme (m). Pour les moyens et les méthodes de MPR en ukrainien nous avons les emprunts de français: патронаж - patronage (m); масаж - massage (m); d'allemand: курорт - Kurort (m). Pour les appareils nous avons les emprunts de français: буж - bougie (f); корсет corset (m); кувез - couveuse (f); лонгета - longuette (f); тампон - tampon (m); бандаж - bandage (m); бювет - buvette (f); компрес - compresse (f); пандус pente (f) douce; d'anglais: еспандер - ехpander; тренування - train; d'allemand: шприц - Spritze (f); de suédois: рибстул / гімнастична стінка - ribbstol. Pour les tests nous avons les emprunts d'anglais: тест - test; скрінінг - screening; тредміл тест (метод діагностики ішемічної хвороби серця) - treadmill test.

\section{La synonymie en MPR}

La synonymie en MPR est causée par l'utilisation des sigles: AVC / accident (m) vasculaire cérébral - порушення мозгового кровообігу; BECS / batterie (f) d'évaluation cognitive et socio-émotionnelle - шкала когнітивної та соціоемоційної оцінки; IMOC / infirmité (f) motrice d'origine cérébrale / paralysie (f) cérébrale - рухова недостатність, спричинена ураженням мозку; CHUP / chaussure (f) thérapeutique à usage permanent - терапевтичне взуття для постійного використання; CMS / capacités (f, pl) de membres supérieurs - оцінка рухових вад верхніх кінцівок у хворих 3 тетраплегією; ECD / échelle (f) de comportement douloureux - шкала больової поведінки; EVF / échelle (f) visuelle de la fatigue - візуальна шкала втоми; EFR / explorations (f, pl) fonctionnelles respiratoires - дослідження функції дихання, спірометрія; ERFC / évaluation (f) rapide des fonctions cognitives - швидка оцінка когнітивних функцій; EFMG / évaluation (f) fonctionnelle motrice globale - функціональна оцінка загальної моторики; des emprunts: courbatures (f, pl) / en anglais "delayed onset muscle soreness" / DOMS - крепатура; enquête (f) / examen (m) / exploration (f) / inspection (f) / investigation (f) / en anglais "check-list" / "check-up" - огляд; mesure (f) de l'indépendance fonctionnelle / MIF / en anglais "Functional independence measure" / FIM - шкала функціональної незалежності; évaluation (f) externe de la sévérité de la dépression chez l'aphasique / en anglais "Aphasia Depression Rating Scale" / ADRS - шкала депресії при афазії; des éponymes: maladie (f) de Down / syndrome (m) de Down / acromicrie (f) congénitale / mongolisme (m) / trisomie (f) 
21 - синдром Дауна / хвороба Дауна / монголізм / трисомія 21; acroparesthésie (f) / chirobrachialgie (f) paresthésique nocturne / maladie (f) de Puthnam акропарестезія (парестезія кінцівок); cage (f) de pouliethérapie / de Rocher каркас для лікування за допомогою системи гирь та блоків; mucoviscidose (f) / fibrose (f) kystique du pancréas / syndrome (m) d'Andersen / triade (f) d'Andersen / MV - муковісцидоз; épreuve (f) de Babinski-Weil / de la déviation angulaire / marche (f) en étoile - клінічний тест на цілісність вестибулярної системи. Nous observons l'utilisation parallèle d'un terme français, d'un emprunt et d'un éponyme: test (m) d'approximation / de compression / de rapprochement / de Volkman / sidelying iliac compression test - тест компресії крижово-клубового суглобу; test (m) d'écartement / test (m) de Verneuil / test (m) en distraction / gapping test / iliac compression test / Ericksen's test - тест крижово-клубового суглобу з розведенням крил клубових кісток. Nous observons le fonctionnement des doublets: cancer (m) mélané / mélanocarcinome $(\mathrm{m})$ / mélanome $(\mathrm{m})$ / nævocancer $(\mathrm{m})$ / nævosarcome $(\mathrm{m})$ / sarcome (m) mélanique / tumeur (m) mélanique II - меланобластома; hydrocéphalie (f) / dilatation (f) ventriculaire / hydropisie (f) du cerveau гідроцефалія / водянка головного мозку; épithélioma (m) / cancer (m) épithelial / carcinome (m) / carcinome (m) cholangiocellulaire - епітеліома / карцинома; traitement (m) par les boues / fangothérapie (f) / illutation (f) / pélothérapie (f) грязелікування; adiposité (f) / adipose (f) / engraissement (m) / obésité (f) / pimélose (f) - ожиріння; coxarthrose (f) / arthrose (f) coxofémorale / arthrose (f) de la hanche / morbus coxae senilis - коксартроз; enflure (f) / gonflement (m) / néoformation (f) / néoplasie (f) / néoplasme ( $\mathrm{m}$ ) / proéminence (f) / tuméfaction (f) / tumeur - пухлина; cyphose (f) / dos (m) rond / dos (m) voûté / gibbosité (f) - кiфоз. Nous observons l'utilisation des dialectismes, des termes familiers et populaires, des homonymes: ambulateur $(\mathrm{m})$ / déambulateur $(\mathrm{m})$ / cadre $(\mathrm{m})$ de marche / gadot $(\mathrm{m})$ / tintébin $(\mathrm{m})$ / tribune (f) / marchette (f) - ходунці; béquille (f) / cabillot (m) / crosse (f) - милиця; acinèse (f) / acinésie (f) / akinésie (f) - акінезія (відсутність активних рухів); agueusie (f) / ageustie (f) - агевзія (втрата смакових відчуттів); dyscinésie (f) / dyskinésie (f) - дискінезія (порушення скоординованих рухових актів).

\section{Discussion}

Les recherches comparatives des différentes terminologies appartiennent aux tendances les plus importantes des études linguistiques modernes. Un terme en tant qu'un mot d'une sphère spéciale de connaissance reflète les résultats de l'expérience et de l'activité pratique de l'homme, fixe les savoirs professionnels et pratiques sur les propriétés de l'objet (Pomirko, 2009).

L'étude de la terminologie de MPR contribue à l'analyse profonde des questions théoriques du développement et des problèmes pratiques de la formation des termes en question en français et en ukrainien. On considère la terminologie de MPR comme un système organisé et structuré des termes. Les problèmes liés à la normalisation de la terminologie de MPR en français et en ukrainien sont conditionnés par le fonctionnement parallèle des abréviations et des termes complets, des éponymes et des toponymes, des emprunts et des homonymes ainsi que des synonymes. La codification de termes doit être complétée par des philologues, des lexicographes et des experts en MPR.

Les deux guerres mondiales ont un impact significatif sur le développement de la médecine physique et de réadaptation, conduisant à un nombre gigantesque de personnes handicapées. Ce fait nous donne la possibilité de considérer que la terminologie de MPR a subi l'évolution la plus intensive pendant le $\mathrm{XX}^{\mathrm{e}}$ siècle, lors de l'élaboration active des appareils et des outils, des moyens, des méthodes et des techniques de réadaptation fonctionnelle, professionnelle et sociale, de rééducation motrice et de récupération des fonctions perdues par les moyens de médecine physique. 
L'un des principaux problèmes aujourd'hui est l'absence des grands dictionnaires français-ukrainien et vice versa de la médecine. En fait, le but fondamental de nos recherches consiste en rédaction de dictionnaire français-ukrainien et vice versa de MPR, où nous planifions de présenter la traduction de cinq groupes de termes -1 ) les professions de MPR; 2) les troubles et les maladies; 3) les tests, les échelles et les classifications en MPR; 4) les moyens et les méthodes de MPR; 5) les abréviations, les sigles et les acronymes de MPR. Chaque terme proposé pour l'inclusion dans le dictionnaire doit être activement utilisé par les spécialistes de MPR et fixé dans les dictionnaires mentionnés déjà. La précision dans la transmission du contenu du concept est particulièrement importante.

Selon la structure de la terminologie de MPR en français et en ukrainien, on distingue les termes simples et les groupes de mots. Pour former les termes de MPR en français et en ukrainien, on utilise activement la dérivation affixale; la composition; les sigles, les abréviations et les acronymes; les emprunts.

Alors, récapitulons nos résultats. Pour nommer une profession de MPR en français on utilise les suffixes -iste, -eur, -iatre et -ier, les éléments d'origine grecque -logue et thérapeute. Nous observons le synonymie des termes créés avec les suffixes -iste / logue. Dans la langue ukrainienne les plus productifs sont: l'élément d'origine grecque -лог, les suffixes $-u \mathrm{~cm} /$-icm, ainsi que l'élément d'origine grecque -терапевт.

Pour former les termes désignant les tests, les échelles et les classifications on utilise le plus souvent les mots: "test" (m) / "épreuve" (f) - "тест"; "échelle" (f) / "score" (m) / "grille" (f) / "batterie" - "шкала"; "classification" (f) - "класифікація" et les mots pour spécifier le test ou la classification. Dans ce groupe en français et en ukrainien on emploie très souvent les emprunts d'anglais.

Les éponymes reflètent les étapes de formation de MPR en tant que science. Nous observons l'utilisation répandue des éponymes pour nommer les troubles et les maladies; les appareils et les méthodes de MPR; les tests, les échelles et les classifications en MPR. En règle générale, on a recours aux éponymes lorsqu'on ne peut pas trouver un terme qui est capable de refléter correctement les signes distinctifs et particuliers d'un phénomène médical et exprimer cela par un ou par quelques mots. Le plus souvent l'éponyme est formé selon le modèle "substantif" + "nom propre" dans le génitif. Presque chaque decouverte d'une nouvelle maladie, des méthodes de MPR conduisent à la formation des éponymes. Quant aux éponymes analysés, ils sont presque tous apparus aux $\mathrm{XIX}^{\mathrm{e}}$ et $\mathrm{XX}^{\mathrm{e}}$ siècles, ce qui, bien sûr, est associé au développement rapide de la médecine en général.

Les sigles, les abréviations et les acronymes sont utilisés pour dénommer les institutions et les organisations; les professions; les troubles et les maladies; les échelles, les tests et les classifications; les moyens et les méthodes de MPR. Un grand nombre des sigles en français qui pour utilisés pour dénommer les associations, les institutions, les comités, les commissions, les syndicats, les fédérations, les organisations; les certificats et les diplômes; les lois montrent clairement que la France accorde une grande attention à la réadaptation des personnes handicapées. En ukrainien les sigles, les abréviations et les acronymes en MPR ne sont pas répandus: ВОO3 / Всесвітня організація охорони здоров'я - OMS / Organisation (f) mondiale de la santé; ХОЗЛ / хронічне обструктивне захворювання легень - ВРСО / broncho-pneumopathie (f) chronique obstructive; ЧСС / частота серцевих скорочень - FC / fréquence (f) cardiaque; MPT / магнітно-резонансна томографія / ядерно-магнітний резонанс - IRM / imagerie (f) par résonance magnétique, mais nécessitent des recherches plus profondes dans l'avenir.

Dans la terminologie française de MPR, le plus grand nombre de termes empruntés vient des langues anglaise et allemande. Dans la terminologie ukrainienne de MPR, on emprunte des langues anglaise, française et italienne. La domination de l'anglais en tant que source principale pour la terminologie de MPR dans les langues que nous 
avons étudiées, s'explique par le développement dynamique de la réadaptation dans les pays anglophones. Le terme emprunté doit correspondre entièrement aux besoins de la terminologie de MPR et aux critères établis par les spécialistes de MPR et par les linguistes. La synonymie des termes de MPR est déterminée par l'utilisation parallèle des abréviations et des formes complètes; des éponymes; des toponymes; des emprunts; des dialectismes; des homonymes; des termes familiers et populaires.

\section{Conclusions}

L'une des principales tâches aujourd'hui est la rédaction des dictionnaires françaisukrainien de MPR ce qui faciliterait la communication entre les spécialistes en MPR, les personnes handicapées, les fonctionnaires et tous ceux qui sont impliqués dans le processus de réadaptation. Les résultats de nos recherches présentent l'importance pratique et théorique pour accomplir ce devoir. On observe les tendances suivantes en français de MPR : l'utilisation étroite des sigles, des abréviations, des acronymes ainsi que des emprunts anglais. Pour la traduction des sigles et des abréviations français en ukrainien, il a fallu chercher les déchiffrements dans les dictionnaires français de MPR, les articles et les travaux scientifiques, utiliser les ressources Internet.

Les compétences professionnelles d'un traducteur-interprète dépendent non seulement de ses connaissances d'un vocabulaire de MPR, mais aussi de son désir d'apprendre et d'approfondir chaque jour son érudition en matière de notions générales de réadaptation. On sait parfaitement que trouver la bonne version d'un terme scientifique pour un traducteur est une priorité. La terminologie ukrainienne de MPR est aujourd'hui en voie de formation et de codification. Elle utilise activement les emprunts anglais et français et les éponymes. Il n'y a pas beaucoup de sigles.

Les perspectives porteront sur l'analyse des termes néologiques, des métaphores ainsi que des enquêtes approfondies concernant les caractéristiques lexicales et sémantiques de la terminologie ukrainienne de MPR et les questions d'approfondissement de compétence communicative des spécialistes en MPR.

\section{Bibliographic references}

BIELIKOVA, N. O. - SUSHCHENKO, L. P. 2009. Terminolohiia napriamu pidhotovky "Zdorovia ludyny", Kyïv, 182 p. (In Ukrainian).

BLOUIN, M. - ECHEVERRI VALLEJO, L. E. 2010. La readaptation [Ressource électronique]. $\quad$ - Available online: http://cirrie.buffalo.edu/encyclopedia/pdf/es/la_rehabilitacion.pdf. (10 Mai 2014) (en français).

BLOUIN, M. 1995. Dictionnaire de la readaptation. Tome 1. Termes techniques d'evaluation: index français-anglais / anglais-français, Sainte-Foy, Quebec, 130 p. (en français).

BLOUIN, M. 1997. Dictionnaire de la readaptation. Tome 2. Termes d'intervention et d'aides techniques: index français-anglais / anglais-français, Sainte-Foy, Quebec, 164 p. (en français).

BOICHUK, T. V. - GOLUBIEVA, M. G. - LEVANDOVSKIY, O. S. 2010. Osnovy diagnostychnykh doslidzen u fizychniy reabilitatsii, Lviv, 240 p. (in Ukrainian).

BOICHUK, T. V. - GOLUBIEVA, M. G. - LEVANDOVSKIY, O. S. 2010. Terminologichniy slovnyk-dovidnyk dlia studentiv spetsialnosti "Zdorovia ludyny" ("Fizychna reabilitatsia"), Ivano-Frankivsk, 150 p. (in Ukrainian).

DE GODEBOUT, J. 1990. Lexique et manuel Anglais-Français pour la Reeducation et la Readaptation, Montpellier, 229 p. (in French and in English).

DUBOGAI, O. D. - ALIOSHYNA, A. I. - LAVRENIUK, V. IE. 2011. Osnovni poniattia i terminy zdoroviazberezhennia ta fizychnoi reabilitatsii $\mathrm{v}$ systemi osvity, Lutsk, 296 p. (in Ukrainian). 
DUBOGAI, O. D. - TKACHUK, A. M. - KOSTIKOVA, S. D. - YEFIMOV, A. O. 1998. Osnovni poniattia i terminy ozdorovchoi fizychnoi kultury ta reabilitatsii, Lutsk, 100 p. (in Ukrainian).

DUFOUR, M. - GEDDA, M. 2007. Dictionnaire de kinesitherapie et readaptation, Paris, 582 p. (en français).

FACIONE-ROGER, J. 2010. Place de la medecine physique et de readaptation dans le soutien sante des forces armees: historique, etat des lieux et perspectives. These pour obtenir le grade de docteur en medecine, Nancy, Universite Henri Poincare, 123 p. (en français).

HAMONET, C. - DE JOUVENCEL, M. - TRONINA-PETIT, J. 2005. Aux origines de la readaptation // In Journal de readaptation medicale. Vol. 25, ${ }^{\circ} 1$. pp. 3-6. DOI: JRM-02-2005-25-1-0242-648X-101019-2005504355. (en français).

HERTSYK, A. M. 2003. Traktuvannia kliuchovykh terminiv v osvitno-profesiinii haluzi fizychnoi reabilitatsii. In Moloda sportyvna nauka Ukrainy, Issue 7, vol. 1, pp. 342-346. (in Ukrainian).

HERTSYK, A. M. 2010. Do pytannia tlumachennia terminiv "fizychna reabilitatsia" ta "fakhivets $\mathrm{z}$ fizychnoi reabilitatsii". In Teoria ta metodyka fizychnoho vykhovannia, no 3, pp. 45-48. (in Ukrainian).

HERTSYK, A. M. 2010a. Fakhivets z fizychnoi reabilitatsii, chy fizychnyi terapevt: natsionalne ta mizhnarodne tlumachennia profesii, In Pedahohika, psykholohia ta medyko-biolohichni problemy fizychnoho vykhovannia, no 10, pp. 21-24. (in Ukrainian).

HERTSYK, A. M. 2016. Vyznachennia poniattia "tekhnolohia" u fizychniy reabilitatsii. In Fizychna aktyvnist, zdorovia i sport, no 1 (23), pp. 32-34. (in Ukrainian).

JANIK, S. - BRUNET, L. - LECOMPTE, L.-L. 1986. Thesaurus a schemas fleches de descripteurs pour l'analyse et la recherche des documents et du materiel avec leurs equivalents en langue anglaise, Quebec, 429 p. (in French and in English).

JANIK, S. 1989. La terminologie du Thesaurus: personne handicapee. In Meta, vol. 34, issue 4, pp. 745-752. DOI: 10.7202/003252ar (en français).

KAMENETZ, H. - KAMENETZ, G. 1972. Dictionnaire de medecine physique, de reeducation et de readaptation fonctionnelles, Paris, 205 p. (en français)

KOVAL, R. S. - TYRAVSKA, O. I. 2017. Naimenuvannia testiv, shkal i klasyfikatsiy u reabilitatsii. In Current issues of Social Studies and History of Medicine. Joint Ukrainian-Romanian scientific journal. Series "Philological sciences", issue 4 (16), pp. 156-160. ISSN 2411-6181 (on-line). ISSN 2311-9896 (print). DOI: 10.24061/2411-6181.4.2017.111 (in Ukrainian).

KOVAL, R. S. - TYRAVSKA, O. I. 2017a. Problemy perekladu terminolohii reabilitatsii. In Visnyk Natsionalnoho Universytetu "Lvivska Politekhnika". Seriia "Problemy ukrainskoi terminolohii", no 869, pp. 39-45. (in Ukrainian). ISSN 03210499

KOVAL, R. S. 2015. Eponimy v terminolohii fizychnoi i medychnoi reabilitatsii (na materiali frantsuzkoi ta ukrainskoi mov). In Naukovyi visnyk Skhidnoievropeiskoho natsionalnoho universytetu imeni Lesi Ukrainky. Seria: Filolohichni nauky, no 3 (304), pp. 293-298. ISSN 1729-360X (in Ukrainian).

KOVAL, R. S. 2015a. Toponimy v terminolohii fizychnoi i medychnoi reabilitatsii. In Naukovyi visnyk Mizhnarodnoho humanitarnoho universytetu. Seriia: Filolohiia, issue 15, vol. 2, pp. 58-60. ISSN 2409-1154 (In Ukrainian).

KOVAL, R. S. 2015b. Zapozychennia v terminolohii reabilitatsii. In Naukovi zapysky Vinnytskoho derzhavnoho pedagogichnoho universytetu imeni Mykhaila Kotsiubynskoho. Seriia: Filolohiia (Movoznavstvo), issue 22, pp. 21-27. (in Ukrainian).

XLinguae, Volume 12 Issue 1XL, January 2019, ISSN 1337-8384, eISSN 2453-711X 125 
KOVAL, R. S. 2016. Abreviatury v terminolohii reabilitatsii. In Aktualni problemy romano-hermanskoi filologii ta prykladnoi linhvistyky, issue 11-12 (part 1), pp. 272276. (In Ukrainian).

KOZYREVA, O. V. - IVANOV, A. A. 2010. Fizicheskaia reabylytatsyia. Lechebnaia fizicheskaia kultura. Kineziterapiia, Moscow, 278 p. (in Russian).

KRAMERS-DE QUERVAIN, I. - KNUSEL, O. 2012. Une specialite "allround" a l'epicentre de la readaptation globale, In Forum Med Suisse, issue 12 (1-2), pp. 20-21 [Ressource électronique]. - Available online: www.rehaschweiz.ch/.../smf_Kramers_2012-01-333_f.pdf (9 Mars 2018) (en français).

KRAVCHENKO, A. I. - LIANNOI, YÜ. O. - KUPYNA, V. V. 2002. Medykobiolohichna ta reabilitatsiina terminolohiia dlia studentiv spetsialnostei "Fizychna reabilitatsiia", "Fizychna kultura", Sumy, 212 p. (in Ukrainian).

LYSENIUK, V. P. - SAMOSIUK, I. Z. - FISENKO L. I. 2007. Medychna reabilitatsia: suchasni standarty, testy, shkaly ta kryterii efektyvnosti. Nyzkointensyvna rezonansna fizioterapia i ii zastosuvannia $\mathrm{v}$ reabilitatsiyniy medycyni, Kyïv, 264 p. (in Ukrainian).

MERZLIKINA, O. A. - HUZII, O. V. 2002. Tlumachnyi slovnyk terminiv i slovospoluchen fizychnoi reabilitatsii. Lviv, 48 p. (In Ukrainian).

MEYER, S. 2013. Ergotherapies. De l'activite a la participation, Paris, 274 p. (en français).

MONET, J. 2003. Emergence de la kinesitherapie en France a la fin du XIX et au debut du XX siecle. Une specialite medicale impossible. Genese, acteurs et interets de 1880 a 1914. These pour le doctorat en sociologie, Paris, 705 p. [Ressource électronique]. - Available online: http://www.bium.univparis5.fr/histmed/asclepiades/pdf/monet1.pdf (9 Mars 2018) (en français).

ORZESHKOVSKIY, V. V. - SAMOSIUK, I. Z. - ORZESHKOVSKIY, VAS. V. Encyklopedicheskiy slovar terminov, upotrebliaemukh $\mathrm{v}$ fizioterapii (fizicheskoi medycyne), Pod red. V. V. Orzeshkovskogo, E. A. Kolesnyka, Kyïv, 2004. 352 p. (in Russian).

POMIRKO, R. S. 2009. Peredmova do monohrafii: Dudok, R. I. 2009. Problemy znachennia ta smyslu termina $v$ humanitarnii sferi, Lviv, 358 p. (in Ukrainian).

PRYSTUPA, YE. N. - VOVKANYCH, A. S. 2011. Rol i misce fakhivtsia z fizychnoi reabilitatsii $\mathrm{v}$ systemi okhorony zdorovia. In Pedahohika, psykholohia ta medykobilohichni problemy fizuchnoho vykhovannia i sportu, pp. 92-96. (in Ukrainian).

WIROTIUS, J.-M. 1999. Histoire de la reeducation. In Encycl Med Chir (Elsevier, Paris), Kinesitherapie-Medecine physique-Readaptation, 26-005-A-10, 25 p. (en français).

WIROTIUS, J.-M. 2011. Les mots de "reeducation", de "readaptation" ont-ils encore leur place en MPR? In Annals of Physical and Rehabilitation Medicine, Vol. 54, $\mathrm{n}^{\circ}$ S1, pp. e282-e283. DOI: 10.1016/j.rehab.2011.07.188 (en français).

WIROTIUS, J.-M. 2012. Quel sens donner a la readaptation? L'apport de la linguistique. In Journal de readaptation medicale, Vol. 32, no 1, pp. 4-18. Doi: 10.1016/j.jrm.2011.11.002 (en français).

ZRIBI, G. - POUPEE-FONTAINE, D. 2011. Dictionnaire du handicap, Rennes: Presses de l'EHESP, 349 p. (en français).

Words: 10187

Characters: 72196 (40,11 standard pages)

Prof. Roman Pomirko, Doctor of Science in Philology, Academician of Academy of Higher Education of Ukraine

Head of French Department of the Foreign Languages Faculty of the Ivan Franko National University of Lviv

Universytetska Str., 1 
79000 Lviv,

Ukraine

Senior lecturer Rostyslav Koval

Department of Ukrainian and Foreign languages

Lviv State University of Physical Culture

Kostiushko Str., 11

79000 Lviv,

Ukraine

kovalrostyslav@gmail.com

Assoc. Prof. Olga Romanchuk, PhD

Head of Department of Ukrainian and Foreign languages

Lviv State University of Physical Culture

Kostiushko Str., 11

79000 Lviv,

Ukraine

olgabrvska@gmail.com

Assoc. Prof. Olesya Cherkhava, Doctor of Science in Philology

Department of English language, The Faculty of Interpreting and Translation

Kyïv National Linguistic University

Velyka Vasylkivska Str., 73

03680 Kyïv-150,

Ukraine

olesya_marchenko@yahoo.com

Assoc. Prof. Nataliia Bazyliak, PhD

Department of Ukrainian and Foreign languages

Lviv State University of Physical Culture

11 Kostiushko Str.

79000 Lviv,

Ukraine

nat.nataly0070@gmail.com 